\begin{tabular}{|c|l|}
\hline Title & On the commutativity of fundamental groups of complements to plane curves \\
\hline Author(s) & SHIMA DA, ICHIRO \\
\hline Citation & $\begin{array}{l}\text { Mathematical Proceedings of the Cambridge Philosophical Society, 123, 49-52 } \\
\text { https://doi.org/10.1017/S0305004197002107 }\end{array}$ \\
\hline Issue Date & 1998 \\
\hline Doc URL & http://hdl.handle.net/2115/5794 \\
\hline Rights & Copyright $\odot$ 1998 Cambridge University Press \\
\hline Type & article \\
\hline File Information & MPCPS123.pdf \\
\hline
\end{tabular}

Instructions for use 


\title{
On the commutativity of fundamental groups of complements to plane curves
}

\author{
BY ICHIRO SHIMADA \\ Department of Mathematics, Faculty of Science, Hokkaido University, Sapporo 060 \\ e-mail: shimada@math.hokudai.ac.jp
}

(Received 14 February 1996; revised 7 June 1996)

\section{Introduction}

In this paper, we prove the following:

Theorem. Let $C \subset \mathbb{P}^{3}$ be a complex reduced irreducible space curve which is nondegenerate (i.e., $C$ is not contained in any plane). Let $p: C \rightarrow \mathbb{P}^{2}$ be a general projection . Then the topological fundamental group $\pi_{1}\left(\mathbb{P}^{2} \backslash p(C)\right)$ is abelian.

When $C$ is non-singular, the image of the general projection has only nodes as its singularities, and hence the complement has an abelian fundamental group thanks to Fulton-Deligne's theorem on Zariski conjecture $([\mathbf{1}, \mathbf{2}])$. The point of our theorem is that we make no assumptions on the singularities of the space curve $C$.

We apply this theorem to the problem of commutativity of fundamental groups of complements to plane curves of a given degree with prescribed numbers of nodes and cusps. Here a cusp means a germ of curve singularity which is analytically isomorphic to a small neighborhood of the singular point of the affine curve $x^{2}+y^{3}=0$. The strongest result concerned with this problem is Nori's result [5, proposition 6.5]. (Nori considered not only plane curves with only nodes and cusps but curves on arbitrary surfaces with any kind of singularities.) Let $C \subset \mathbb{P}^{2}$ be a reduced irreducible plane curve of degree $d$ whose singular locus consists of $n$ nodes and $k$ cusps.

Theorem (Nori). Suppose that $2 n+6 k<d^{2}$. Then $\pi_{1}\left(\mathbb{P}^{2} \backslash C\right)$ is abelian.

We will prove a proposition which enlarges slightly the region of $(d, n, k)$ on which the commutativity of $\pi_{1}\left(\mathbb{P}^{2} \backslash C\right)$ is guaranteed.

Proposition 1. Suppose that $2 n \geqslant d^{2}-5 d+8$. Then $\pi_{1}\left(\mathbb{P}^{2} \backslash C\right)$ is abelian.

\section{Proof of Theorem}

Let $G$ denote the Grassmannian variety Grass $\left(\mathbb{P}^{1}, \mathbb{P}^{3}\right)$ of all lines in $\mathbb{P}^{3}$, and let $\left(\mathbb{P}^{3}\right)^{*}$ be the dual projective space Grass $\left(\mathbb{P}^{2}, \mathbb{P}^{3}\right)$ of $\mathbb{P}^{3}$. For a plane $\Pi \in\left(\mathbb{P}^{3}\right)^{*}$, we put

$$
\Sigma(\Pi):=\{l \in G ; l \subset \Pi\} .
$$

We also put

$$
U:=\{l \in G ; C \cap l=\theta\} .
$$

When $\Pi \in\left(\mathbb{P}^{3}\right)^{*}$ is general, no three points among $C \cap \Pi$ are co-linear. Indeed, let $B \subset\left(\mathbb{P}^{3}\right)^{*}$ be the Zariski open dense subset consisting of the planes which intersect $C$ 
at its non-singular points transversely. Let $\Pi_{0} \in B$ be a plane which serves as a base point of $B$. Since $C$ is irreducible, the monodromy group of the action of $\pi_{1}\left(B, \Pi_{0}\right)$ on the set $C \cap \Pi_{0}$ is the full symmetric group, because the action is 2-transitive and the monodromy group contains a simple transposition (ef. [4, Uniform Position Lemma]). Hence if there exist co-linear three points among $C \cap \Pi$ for a general $\Pi \in$ $\left(\mathbb{P}^{3}\right)^{*}$, then all points in $C \cap \Pi$ are on a line for a general $\Pi$. Note that this condition is closed for the choice of $\Pi$ in $\left(\mathbb{P}^{3}\right)^{*}$ because $C$ is non-degenerate. Thus all points in $C \cap \Pi$ are on a line for every $\Pi$, which is absurd. Consequently, $\Sigma(\Pi) \backslash U$ is a union of lines on $\Sigma(\Pi) \cong \mathbb{P}^{2}$, which is a normal crossing divisor when the plane $\Pi$ is generally chosen. Hence $\pi_{1}(\Sigma(\Pi) \cap U)$ is abelian for a general $\Pi$ because of Fulton-Deligne's theorem ([1-3]). Let $I \subset\left(\mathbb{P}^{3}\right)^{*} \times G$ be the closed subvariety $\{(\Pi, l) ; \Pi \supset l\}$. The second projection $\rho_{2}: I \rightarrow G$ is a fibre bundle with fibres isomorphic to $\mathbb{P}^{1}$. Hence $\rho_{2}$ induces an isomorphism $\pi_{1}\left(\rho_{2}^{-1}(U)\right) \cong \pi_{1}(U)$. Consider the projection $\rho_{1}: \rho_{2}{ }^{-1}(U) \rightarrow$ $\left(\mathbb{P}^{3}\right)^{*}$. The fibre $\rho_{1}^{-1}(\Pi) \subset \rho_{2}^{-1}(U)$ over $\Pi \in\left(\mathbb{P}^{3}\right)^{*}$ is isomorphic to $\Sigma(\Pi) \cap U$, which is non-empty and non-singular for every $\Pi$. We apply Nori's Lemma [5, lemma 1.5 (C)] to conclude that the inclusion of the fibre induces a surjection $\pi_{1}(\Sigma(\Pi) \cap U) \rightarrow$ $\rightarrow \pi_{1}\left(\rho_{2}^{-1}(U)\right)$ for a general $\Pi$. Hence $\pi_{1}\left(\rho_{2}^{-1}(U)\right)$ is abelian, and thus $\pi_{1}(U)$ is also abelian.

We fix a plane $\Pi_{0} \subset \mathbb{P}^{3}$ and denote by $A$ the complement $\mathbb{P}^{3} \backslash \Pi_{0}$. Let $J_{A} \subset A \times G$ be the closed subvariety $\{(P, l) ; P \in l\}$. Consider the projection $\tau_{2}: J_{A} \rightarrow G$. If $l \notin \Pi_{0}$, then $\tau_{2}^{-1}(l)$ is isomorphic to $\mathbb{A}^{1}$, while if $l \subset \Pi_{0}$, then $\tau_{2}^{-1}(l)$ is empty. Thus $\tau_{2}$ induces an isomorphism $\pi_{1}\left(\tau_{2}^{-1}(U)\right) \cong \pi_{1}\left(U \backslash \Sigma\left(\Pi_{0}\right)\right)$. Since $\Sigma\left(\Pi_{0}\right)$ is of codimension 2 in $G$, we have $\pi_{1}\left(U \backslash \Sigma\left(\Pi_{0}\right)\right) \cong \pi_{1}(U)$. Therefore, $\pi_{1}\left(\tau_{2}^{-1}(U)\right)$ is isomorphic to $\pi_{1}(U)$. We have an isomorphism $J_{A} \cong A \times \Pi_{0}$ given by $(P, l) \mapsto\left(P, l \cap \Pi_{0}\right)$. Let $\tilde{\tau}_{2}: A \times \Pi_{0} \rightarrow G$ be the morphism corresponding to $\tau_{2}: J_{A} \rightarrow G$ via this isomorphism. Let $D \subset G$ be the Zariski closed subset $G \backslash U$. We provide it with the reduced structure. For $P \in A$, consider the scheme theoretic intersection

$$
D_{P}:=\left(\{P\} \times \Pi_{0}\right) \cap \tilde{\tau}_{2}^{-1}(D),
$$

which is considered as a sub-scheme of $\Pi_{0}$. If $P \in C$, then $D_{P}=\Pi_{0}$. Suppose that $P \notin C$. Let $p\langle P\rangle: C \rightarrow \Pi_{0}$ denote the projection with the centre $P$. Then we see that the reduced part $\left(D_{P}\right)_{\text {red }}$ of $D_{P}$ coincides with the reduced part $p\langle P\rangle(C)_{\text {red }}$ of the image of the projection $p\langle P\rangle$. Hence $D_{P}$ is a reduced divisor of $\Pi_{0}$ if $\operatorname{deg} p\langle P\rangle(C)=$ $\operatorname{deg} C$.

Claim. There are at most finitely many points $P \in A \backslash C$ such that the degree of $p\langle P\rangle(C)_{\text {red }}$ is less than the degree of $C$.

Since $C \cap A \subset A$ is of codimension 2, Claim implies that the locus of all points $P \notin A$ such that the scheme theoretic intersection $D_{P}$ is not a reduced divisor of $\Pi_{0}$ is of codimension 2 in $A$. Let $P \in A$ be generally chosen. Applying [6, theorem 1], we get $\pi_{1}\left(\Pi_{0} \backslash p\langle P\rangle(C)\right) \cong \pi_{1}\left(\tilde{\tau}_{2}^{-1}(U)\right) \cong \pi_{1}(U)$. Therefore $\pi_{1}\left(\Pi_{0} \backslash p\langle P\rangle(C)\right)$ is abelian, and Theorem is proved.

Proof of Claim. Suppose that there exists an irreducible curve $\Xi \subset A \backslash C$ such that $\operatorname{deg} p\langle P\rangle(C)_{\text {red }}<\operatorname{deg} C$ for all $P \in \Xi$. Let $P_{0}$ be a general point of $\Xi$ and $Q_{0}$ a general point of $C$. Since $P_{0}$ is not on $C$, the projection $p\left\langle P_{0}\right\rangle: C \rightarrow \Pi_{0}$ with the centre $P_{0}$ must be of mapping degree $\geqslant 2$ onto its image. Thus there exists a point $R_{0} \in C$ such 
that $Q_{0} \neq R_{0}$ and $p\left\langle P_{0}\right\rangle\left(Q_{0}\right)=p\left\langle P_{0}\right\rangle\left(R_{0}\right)$. Since $Q_{0}$ is chosen generally, $p\left\langle P_{0}\right\rangle\left(Q_{0}\right)$ is a non-singular point of $p\left\langle P_{0}\right\rangle(C)_{\text {red }}$, and the morphism $p\left\langle P_{0}\right\rangle: C \rightarrow p\left\langle P_{0}\right\rangle(C)_{\text {red }}$ is étale at $Q_{0}$ and at $R_{0}$. This implies that the tangent lines $l\left(Q_{0}\right)$ and $l\left(R_{0}\right)$ of $C$ at $Q_{0}$ and at $R_{0}$, respectively, intersect each other in $\mathbb{P}^{3}$. Since $P_{0}$ is also chosen generally on $\Xi$, there exist small open neighbourhoods (in the sense of complex analytic geometry) $V \subset \Xi$ of $P_{0}$ in $\Xi$ and $W \subset C$ of $R_{0}$ in $C$, and an isomorphism $f: V \stackrel{\sim}{\longrightarrow} W$ such that $p\langle P\rangle\left(Q_{0}\right)=p\langle P\rangle(f(P))$ for all $P \in V$. This implies that the tangent line $l(R)$ of $C$ at $R$ intersects $l\left(Q_{0}\right)$ for all $R \in W$. Therefore $l(R) \cap l\left(Q_{0}\right) \neq \theta$ for every non-singular point $R$ of $C$. However, consider the projection $\lambda: C \rightarrow \mathbb{P}^{1}$ with the centre $l\left(Q_{0}\right)$. Since $C$ is non-degenerate, $\lambda$ is surjective, and hence, by Sard's theorem, $\lambda$ is smooth at a general point $R \in C$. This implies that $l(R) \cap l\left(Q_{0}\right)=\theta$ for a general $R \in C$, and we get a contradiction.

\section{Proof of Proposition 1}

First note that if $2 n \geqslant d^{2}-5 d+8$ and $2 n+2 k<d^{2}-4 d+3$, then $2 n+6 k$ is automatically less than $d^{2}$. Therefore, taking Nori's theorem into account, we may assume that the inequality $2 n+2 k \geqslant d^{2}-4 d+3$ also holds.

Let $\nu: \tilde{C} \rightarrow C$ be the normalization of $C$. The genus of $\tilde{C}$ is given by

$$
g=(d-1)(d-2) / 2-n-k .
$$

The assumed inequalities imply that

$$
d \geqslant \max (2 g+1, g+3), \quad \text { and } \quad k \leqslant d-g-3 .
$$

Let $L$ be the pull-back of $\mathcal{O}_{C}(1)$ by $\nu$, which is an invertible sheaf of degree $d$ on $\tilde{C}$. By the first inequality of $(2 \cdot 1), L$ is very ample and $\operatorname{dim}|L|=d-g$. Let $C^{\prime}$ be the image of the embedding of $\tilde{C}$ in $\mathbb{P}^{d-g}$ by $L$. Then $C$ is obtained by a certain projection $C^{\prime} \rightarrow \mathbb{P}^{2}$. Let $\Gamma \subset \mathbb{P}^{d-g}$ be the centre of this projection, which is a linear subspace of codimension 3. Let $T \subset \mathbb{P}^{d-g}$ be the union of all lines tangent to $C^{\prime}$. Then $\Gamma$ intersects $T$ at distinct $k$ points $P_{1}, \ldots, P_{k}$, each of which corresponds to a cusp of $C$ bijectively. By the second inequality of $(2 \cdot 1)$, there exists a linear subspace $\Lambda \subset \Gamma$ of codimension 1 which contains $P_{1}, \ldots, P_{k}$. Let $p\langle\Lambda\rangle: C^{\prime} \rightarrow \mathbb{P}^{3}$ be the projection with the centre $\Lambda$. The image $C^{\prime \prime} \subset \mathbb{P}^{3}$ is a non-degenerate curve, which has at least $k$ singular points $Q_{1}, \ldots, Q_{k}$ corresponding to $P_{1}, \ldots, P_{k} \in \Lambda \cap T$. The projection $p\langle\Gamma\rangle: C^{\prime} \rightarrow C$ with the centre $\Gamma$ factors as $C^{\prime} \rightarrow C^{\prime \prime} \rightarrow C$, and $C^{\prime \prime} \rightarrow C$ maps $Q_{1}, \ldots$, $Q_{k}$ to the cusps of $C$ bijectively. Therefore, $C^{\prime \prime}$ has $k$ cusps and some nodes (possibly none) as its only singularities. Thus the image $p\left(C^{\prime \prime}\right)$ of the general projection of $C^{\prime \prime}$ to $\mathbb{P}^{2}$ has the same numbers and types of singular points as the original curve $C$. Note that $C$ and $p\left(C^{\prime \prime}\right)$ are contained in an irreducible equisingular family of plane curves, every member of which is obtained as an image of a projection from $C^{\prime \prime} \subset \mathbb{P}^{3}$ to $\mathbb{P}^{2}$. Thus our main theorem implies that $\pi_{1}\left(\mathbb{P}^{2} \backslash C\right)$ is abelian.

\section{Examples}

Let $\mathscr{A}$ denote the set of triples $(d, n, k)$ with $d \in \mathbb{Z}_{>0}, n \in \mathbb{Z}_{\geqslant 0}$ and $k \in \mathbb{Z}_{\geqslant 0}$ for which the following hold:

(i) There exists a reduced irreducible plane curve $C \subset \mathbb{P}^{2}$ of degree $d$ with $n$ nodes and $k$ cusps as its only singularities. 
(ii) For any plane curve $C$ as in (i), $\pi_{1}\left(\mathbb{P}^{2} \backslash C\right)$ is abelian.

Nori's result says that if $(d, n, k)$ satisfies (i) and $2 n+6 k<d^{2}$, then $(d, n, k) \in \mathscr{A}$. Using Proposition 1, we shall enlarge the known region of $\mathscr{A}$.

Let $C \subset \mathbb{P}^{n}$ be a non-degenerate irreducible curve of degree $d$ whose singular locus consists of only nodes and cusps. Let $k$ be the number of cusps. We write by $\operatorname{Tan}(C) \subset \mathbb{P}^{n}$ the union of all lines in $\mathbb{P}^{n}$ tangent to $C$ at its non-singular points. The following proposition is elementary:

Proposition 2. If $P$ is a general point of Tan $(C)$, then the image of the projection $C \rightarrow \mathbb{P}^{n-1}$ with the centre $P$ is of degree $d$, has only nodes and cusps as its singularities, and the number of cusps is $k+1$.

Let $C$ be a non-singular curve of genus $g$, and $L$ a line bundle on $C$ of degree $d \geqslant \max (2 g+1, g+3)$. We embed $C$ into $\mathbb{P}^{d-g}$ by $|L|$. Applying Proposition 2 to $C \subset \mathbb{P}^{d-g}$ repeatedly, we obtain a non-degenerate space curve $C^{\prime} \subset \mathbb{P}^{3}$ of degree $d$ with certain number of nodes (possibly none) and $d-g-3$ cusps as its only singularities. Let $C^{\prime \prime} \subset \mathbb{P}^{2}$ be the image of a general projection $C^{\prime} \rightarrow \mathbb{P}^{2}$. Then $C^{\prime \prime}$ is an irreducible plane curve of degree $d$ with

$$
n=\left(d^{2}-5 d+8\right) / 2 \text { nodes and } k=d-g-3 \text { cusps }
$$

as its only singularities. Since $2 n \geqslant d^{2}-5 d+8$, Proposition 1 implies that $(d, n, k) \in$ $\mathscr{A}$. Thus, choosing a line bundle of degree $\geqslant 6 g+10$, we obtain a region of $\mathscr{A}$ not covered by Nori's theorem.

Note that there is another known region of $\mathscr{A}$, which is given by the following theorem due to Zariski [7]. Let $R(d, k) \subset \mathbb{P}_{*}\left(\Gamma\left(\mathbb{P}^{2}, \mathcal{O}(d)\right)\right)$ be the locus of all rational plane curves $C$ of degree $d$ with $n$ nodes and $k$ cusps as its only singularities. Since the genus of the normalization of $C$ is 0 , we have $n=(d-1)(d-2) / 2-k$.

Theorem (Zariski). (1) The locus $R(d, k)$ is non-empty if and only if $k \leqslant 3(d-2) / 2$. (2) For a member $C$ of $R(d, k), \pi_{1}\left(\mathbb{P}^{2} \backslash C\right)$ is abelian unless $d$ is even and $k=3(d-2) / 2$.

Thus, if $n+k=(d-1)(d-2) / 2$ and $k \leqslant(3 d-7) / 2$, we have $(d, n, k) \in \mathscr{A}$.

Acknowledgement. I would like to thank Max-Planck-Institut für Mathematik in Bonn for providing me with the stimulating research environment. This work was done during my stay at the Institute in 1995.

\section{REFERENCES}

[1] P. Deligne. Le groupe fondamental du complément d'une courbe plane n'ayant que des points doubles ordinaires est abélien. Sém. Bourbaki, no. 543, 1979/80, Lecture Notes in Math. vol. 842 (Springer-Verlag 1981), 1-10.

[2] W. Fulton. On the fundamental group of the complement of a node curve. Ann. Math. 111 (1980), 407-409.

[3] W.Fulton and R. Lazarsfeld. Connectivity and its applications in algebraic geometry. Lecture Notes in Math., vol. 862 (Springer-Verlag, 1981), 26-92.

[4] J. Harris. The genus of space curves. Math. Ann. 249 (1980), 191-204.

[5] M. Nori. Zariski's conjecture and related problems. Ann. Sci. École Norm. Sup. (4), 16 (1983), $305-344$

[6] I. Shimada. Fundamental groups of open algebraic varieties. Topology, 34 (1995), 509-532.

[7] O. Zariski. On the Poincaré group of rational plane curves. Amer. J. Math., 58 (1936), 607-619; Collected Papers, Volume 3 (The MIT Press, 1978), 266-278. 